\title{
q-Oscillator Algebra And d-Orthogonal Polynomials
}

Fethi Bouzeffour, Ali Zagouhani

To cite this article: Fethi Bouzeffour, Ali Zagouhani (2013) $q$-Oscillator Algebra And $d$ Orthogonal Polynomials, Journal of Nonlinear Mathematical Physics 20:4, 480-494, DOI: https://doi.org/10.1080/14029251.2013.868262

To link to this article: https://doi.org/10.1080/14029251.2013.868262

Published online: 04 January 2021 


\title{
$q$-Oscillator Algebra And $d$-Orthogonal Polynomials
}

\author{
Fethi Bouzeffour \\ Department of Mathematics, College of Sciences \\ King Saud University, P. O Box 2455 Riyadh 11451, Saudi Arabia \\ fbouzaffour@ksu.edu.sa \\ Ali Zagouhani \\ Département de Mathématiques, IPEI de Bizerte, \\ Univertsité de Carthage, 7021 Zarzouna, Tunisia \\ ali.zaghouani@gmail.com
}

Received 27 February 2013

Accepted 13 August 2013

\begin{abstract}
In this paper we express the matrix coefficients of the Fock representation of a $q$-oscillator algebra in terms of the $d$-orthogonal Al-Salam Carlitz polynomials. Also, we derive a generating functions, recurrence relations and $q$-difference equations for these $d$-orthogonal polynomials.

Keywords: Coherent states; Quantum algebra; Basic orthogonal polynomials.

2000 Mathematics Subject Classification: 81R30, 81R60, 33D52
\end{abstract}

\section{Introduction}

Let $\mathscr{P}$ be the linear space of polynomials with complex coefficients and let $\mathscr{P}^{\prime}$ be its algebraic dual. A polynomials sequence $\left\{P_{n}\right\}_{n}$ is called a polynomial set if and only if $\operatorname{deg}\left(P_{n}\right)=n$ for all nonnegative integer $n$. We denote by $\langle u, f\rangle$ the effect of the linear functional $u \in \mathscr{P}^{\prime}$ on the polynomial $f \in \mathscr{P}$.

Let $\left\{P_{n}\right\}_{n}$ be a polynomials set in $\mathscr{P}$. The corresponding dual sequence $\left(u_{n}\right)$ is defined by

$$
<u_{n}, P_{m}>=\delta_{n m}, n, m=0,1, \ldots,
$$

where $\delta_{n m}$ being the Kronecker symbol.

A natural extension of the notion of orthogonality was introduced by Van Iseghem [7] and Maroni 
[15] as follows:

Let $d$ be a positive integer and let $\left\{P_{n}\right\}_{n}$ be a polynomials set in $\mathscr{P}$.

$\left\{P_{n}\right\}_{n}$ is called a $d$-orthogonal polynomials set $(d$-OPS for shorter) with respect to the $d$-dimensional functional vector $\mathscr{U}={ }^{t}\left(u_{0}, u_{1}, \ldots, u_{d-1}\right)$ if it verifies the following conditions:

$$
\begin{gathered}
\left\langle u_{k}, P_{m} P_{n}\right\rangle=0, n>m d+k+1, \\
\left\langle u_{k}, P_{n} P_{n d+k}\right\rangle \neq 0, \quad n \geq 0 .
\end{gathered}
$$

For each integer $k \in\{0,1, \ldots, d-1\}$.

For the particular case $d=1$, we meet the well known notion of orthogonality.

Recall that $\left\{P_{n}\right\}_{n}$ is $d$-OPS if and only if it satisfies a recurrence relation of order $d+1$ of the type

$$
x P_{n}(x)=\beta_{n+1} P_{n+1}(x)-\sum_{k=0}^{d} \alpha_{k, n-k} P_{n-k}(x),
$$

where $\beta_{n+1} \alpha_{0, n-d} \neq 0$ and the convention $P_{-n}=0, n \geq 1$. The result for $d=1$ is reduced to the so-called Favard Theorem. During the past two decades, the $d$-OPS have been the subject of numerous investigations and applications. In particular they are connected with the study of vector padé approximants, simultaneous padé approximants and other problems such as vectorial continued fractions and polynomials solutions of the higher order differential equations. We mention also the appearance of multiple orthogonal polynomials is some problems of modern mathematical physics. The $d$-OPS can be obtained from general multiple orthogonal polynomials under some restrictions upon their parameters [1]. We mention also that numerous explicit examples of such polynomials have "good properties" that's to say explicit expression in terms of generalized hypergeometric functions or possessing some "classical properties" (see, [15]). A new applications of the $d$-OPS was presented recently in [17] by L.vinet and A.Zhedanov is connected with nonlinear automorphisms of the Weyl algebra.

In the same context, we would like to present a $q$-analogue of this work. In fact, we will consider an operator $S$ which is no longer unitary and the corresponding matrix coefficients of this operator with respect to the initial basis give arise to a system of polynomials, which essentially coincides with a $q$-Charlier polynomials $d$-OPS. We show that almost all nontrivial properties the $d$-OPS $q$ Charlier polynomials can be derived directly from their definition as matrix elements of the Fock representation of the $q$-oscillator algebra.

\section{The $q$-Oscillator algebra}

In this section we consider a form of the $q$-oscillator algebra and we discuss some of its basic properties. Let us first review a few basic notions of $q$-calculus; the interested reader may consult [5]. Let $q$ be a real number $0<q<1$. The $q$-shifted factorial are defined by

$$
\begin{aligned}
& (a ; q)_{n}:=\prod_{k=0}^{n-1}\left(1-a q^{k}\right), \\
& \left(a_{1}, \ldots, a_{r} ; q\right)_{n}:=\left(a_{1} ; q\right)_{n} \ldots\left(a_{r} ; q\right)_{n}, n=0,1, \ldots
\end{aligned}
$$


We denote also

$$
\left[\begin{array}{l}
n \\
k
\end{array}\right]_{q}:=\frac{(q ; q)_{n}}{(q, q)_{k}(q ; q)_{n-k}} .
$$

The $q$-exponentials functions are defined by [5]

$$
\begin{aligned}
& e_{q}(z):=\sum_{n=0}^{\infty} \frac{z^{n}}{[n]_{q} !}=\frac{1}{((1-q) z ; q)_{\infty}},|z|<\frac{1}{1-q}, \\
& E_{q}(z):=\sum_{n=0}^{\infty} q^{n(n-1) / 2} \frac{z^{n}}{[n]_{q} !}=(-(1-q) z ; q)_{\infty}, z \in \mathbb{C},
\end{aligned}
$$

where

$$
(a ; q)_{\infty}:=\prod_{k=0}^{\infty}\left(1-a q^{k}\right)
$$

and

$$
[n]_{q}=\frac{1-q^{n}}{1-q}, \quad[n]_{q} !=\prod_{k=1}^{n}[k]_{q}
$$

The $q$-difference operator

$$
D_{q} f(x)=\frac{f(x)-f(q x)}{(1-q) x}
$$

We have

$$
D_{q}(f g)(x)=g(q x) D_{q} f(x)+f(x) D_{q} g(x) .
$$

\subsection{The Fock representations of the q-Oscillator algebra}

In the literature there are several forms of the $q$-deformed oscillator algebra, see [11, Ch.5]. In this work, we consider the $q$-oscillator algebra denoted by $\mathscr{A}_{A}$, which is the associative algebra over $\mathbb{C}$ generated by $A_{-}, A_{+}, q_{0}^{A}, q^{-A_{0}}$ and relations (see [11])

$$
\left[A_{-}, A_{+}\right]_{q}=1, q^{A_{0}} A_{+}=q A_{+} q^{A_{0}}, q^{A_{0}} A_{-}=q^{-1} A_{-} q^{A_{0}}, q^{A_{0}} q^{-A_{0}}=q^{-A_{0}} q^{A_{0}}=1 .
$$

where

$$
[A, B]_{q}:=A B-q B A .
$$

In the case $q=1$, this algebra represents the one-dimensional harmonic oscillator algebra generated by three generators $a, a^{*}$ and 1 with relations

$$
a a^{*}-a^{*} a=1,1 a=a 1,1 a^{*}=a^{*} 1 .
$$

Let $\mathscr{H}$ the be the Hilbert space with orthonormal basis $\{\mid n>\}_{n \in \mathbb{N}}$ and let $\mathscr{D}$ be the linear dense subspace of $\mathscr{H}$ spanned by $\{\mid n>\}_{n \in \mathbb{N}}$. Here we have used the standard Dirac notation (see [16]). 
In this notation an state $|\psi\rangle$ has the decomposition

$$
\left.\left|\psi>=\sum_{n=0}^{\infty}\langle n \mid \psi\rangle\right| n\right\rangle,
$$

where $\langle n \mid \psi\rangle$ means the scalar product of the two states $|n\rangle$ and $|\varphi\rangle$.

The Fock representation of the $q$-oscillator algebra $\mathscr{A}_{A}$ is given by

$$
\begin{aligned}
& A_{+}\left|n>=\sqrt{[n+1]_{q}}\right| n+1>, \\
& A_{-}\left|n>=\sqrt{[n]_{q}}\right| n-1>, \\
& q^{A_{0}}\left|n>=q^{n}\right| n>.
\end{aligned}
$$

From (2.5) we get

$$
\left|n>=\frac{A_{+}^{n}}{\sqrt{[n]_{q} !}}\right| 0>,
$$

where the vector $\mid 0>$ is normalized by the condition

$$
A_{-} \mid 0>=0 .
$$

It's clear that from (2.5) and (2.6) the operator $A_{+} A_{-}$is hermitian and has for $n=0,1, \ldots$, the $q$-numbers $[n]_{q}$ as eigenvalues

$$
A_{+} A_{-}\left|n>=[n]_{q}\right| n>.
$$

We denote by $\mid z>$ the $q$-coherent state defined by

$$
\left|z>=e_{q}\left(z A_{+}\right)\right| 0>=\sum_{n=0}^{+\infty} \frac{z^{n}}{\sqrt{[n] !_{q}}} \mid n>.
$$

The state $\mid z>$ can be looked upon as an eigenstate of the operator $A_{-}$such that

$$
A_{-}|z>=z| z>\text {. }
$$

For $q$-coherent states $\mid z_{1}>$ and $\mid z_{2}>$, we have

$$
<z_{1} \mid z_{2}>=e_{q}\left(z_{1} \overline{z_{2}}\right) .
$$

In addition, if

$$
\psi(z)=<\bar{z} \mid \psi>
$$

then

$$
D_{q} \psi(z)=<z\left|A_{-}\right| \psi>\text { and } z \psi(z)=<z\left|A_{+}\right| \psi>\text {. }
$$

Let $S\left(A_{-}, A_{+}, A_{0}\right)$ be an operator constructed from operators $A_{-}, A_{+}, A_{0}$. We assume that this operator is invertible, i.e there exists an operator $S^{-1}\left(A_{-}, A_{+}, A_{0}\right)$ such that

$$
S S^{-1}=S^{-1} S=1 \text {. }
$$


Consider two systems of matrix coefficients:

$$
\psi_{n k}=<k|S| n>\text { and } \phi_{n k}=<n\left|S^{-1}\right| k>.
$$

It is assumed that the functions $\psi_{n k}$ and $\phi_{n k}$ do exist. A simple computation shows that

$$
S^{-1}\left|n>=\sum_{k=0}^{\infty}<k\right| S^{-1}|n>| k>
$$

and

$$
S S^{-1}\left|n>=\sum_{k=0}^{\infty} \sum_{r=0}^{\infty}<k\right| S^{-1}|n><r| S|k>| r>.
$$

Then

$$
<m\left|S S^{-1}\right| n>=\sum_{k=0}^{\infty}<m|S| k><k\left|S^{-1}\right| n>,
$$

and by (2.11) we obtain the identities

$$
\sum_{k=0}^{\infty}<m|S| k><k\left|S^{-1}\right| n>=<m\left|S S^{-1}\right| n>=<m \mid n>=\delta_{m n} .
$$

Similarly,

$$
\sum_{n=0}^{\infty}<k\left|S^{-1}\right| n><n|S| s>=<k\left|S^{-1} S\right| s>=<k \mid s>=\delta_{k s} .
$$

Hence, the matrix elements $\psi_{n k}, \phi_{n k}$ satisfy the bi-orthogonality relations

$$
\sum_{k=0}^{\infty} \psi_{k n} \phi_{k m}=\delta_{m n} \text { and } \sum_{n=0}^{\infty} \psi_{s n} \phi_{k n}=\delta_{k s}
$$

\subsection{Identities in q-oscillator algebra}

The theory of quantum algebra and in particular $q$-oscillator algebra has been successful in producing identities for $q$-special functions (see [14]) and further references given there. From [14, Proposition 3.1] we have

$$
\begin{aligned}
& e_{q}\left(q^{A_{0}}+A_{+}\right)=e_{q}\left(A_{+}\right) e_{q}\left(q^{A_{0}}\right), E_{q}\left(q^{A_{0}}+A_{+}\right)=E_{q}\left(q^{A_{0}}\right) E_{q}\left(A_{+}\right), \\
& e_{q}\left(A_{-}+q^{A_{0}}\right)=e_{q}\left(q^{A_{0}}\right) e_{q}\left(A_{-}\right), E_{q}\left(A_{-}+q^{A_{0}}\right)=E_{q}\left(A_{-}\right) E_{q}\left(q^{A_{0}}\right) .
\end{aligned}
$$

Proposition 2.1. For $n=0,1,2, \ldots$, we have

$$
\begin{aligned}
& {\left[A_{-}, A_{+}^{n}\right]=[n]_{q} A_{+}^{n-1} q^{A_{0}},} \\
& {\left[A_{-}^{n}, A_{+}\right]=[n]_{q} q^{A_{0}} A_{-}^{n-1} .}
\end{aligned}
$$

Moreover, if $f(z)=\sum_{n=0}^{\infty} a_{n} z^{n}$ is a formal power series, we have

$$
\left[A_{-}, f\left(A_{+}\right)\right]=D_{q} f\left(A_{+}\right) q^{A_{0}},\left[f\left(A_{-}\right), A_{+}\right]=q^{A_{0}} D_{q} f\left(A_{-}\right) .
$$


F. Bouzeffour \& A. Zaghouani

Proof. Let $n \in \mathbb{N}$, from (2.4) we have

$$
\begin{aligned}
{\left[A_{-}^{n}, A_{+}\right] } & =\sum_{i=0}^{n-1} A_{-}^{i}\left[A_{-}, A_{+}\right] A_{-}^{n-1-i} \\
& =\sum_{i=0}^{n-1} A_{-}^{i} q^{A_{0}} A_{-}^{n-1-i} \\
& =\sum_{i=0}^{n-1} q^{i} q^{A_{0}} A_{-}^{n-1} \\
& =[n]_{q} q^{A_{0}} A_{-}^{n-1} .
\end{aligned}
$$

The identity (2.16), follows from (2.14) and (2.15) and the fact that

$$
\left[A_{+}, f\left(A_{-}\right)\right]=\sum_{n \geq 0} a_{n}\left[A_{-}^{n}, A_{+}\right] \text {and }\left[f\left(A_{-}\right), A_{+}\right]=\sum_{n \geq 0} a_{n}\left[A_{-}^{n}, A_{+}\right] .
$$

Proposition 2.2. Let $P$ be a polynomial and t a complex number, we have

$$
\begin{aligned}
& e_{q}\left(t A_{-}\right) P\left(A_{+}\right) E_{q}\left(-t A_{-}\right)=P\left(A_{+}+t q^{A_{0}}\right), \\
& E_{q}\left(t A_{+}\right) P\left(A_{-}\right) e_{q}\left(-t A_{+}\right)=P\left(A_{-}-t q^{A_{0}}\right) .
\end{aligned}
$$

Proposition 2.3. Let $N \geq 0$. For all complex numbers $a_{0}, \ldots, a_{N}$, we have

$$
\begin{aligned}
& \prod_{i=0}^{N} e_{q}\left(a_{i} A_{-}\right) A_{+} A_{-} \prod_{i=0}^{N} E_{q}\left(-a_{i} A_{-}\right)=q^{A_{0}}\left[1-\prod_{i=0}^{N}\left(1-a_{i} A_{-}\right)\right]+A_{+} A_{-} \\
& \prod_{i=0}^{N} e_{q}\left(a_{i} A_{-}\right) q^{A_{0}} \prod_{i=0}^{N} E_{q}\left(-a_{i} A_{-}\right)=q^{A_{0}} \prod_{i=0}^{N}\left(1-a_{i}(1-q) A_{-}\right) .
\end{aligned}
$$

Proof. We will prove the formula (2.18) by recurrence.

For $N=0$, we have

$$
e_{q}\left(a_{0} A_{-}\right) A_{+} E_{q}\left(-a_{0} A_{-}\right)=a_{0} q^{A_{0}} A_{-}+A_{+} A_{-} .
$$

We suppose that this expression is true for $N$, stay it true for the order $N+1$ ?

We have

$$
\begin{aligned}
\prod_{i=0}^{N+1} e_{q}\left(a_{i} A_{-}\right) A_{+} \prod_{i=0}^{N+1} E_{q}\left(-a_{i} A_{-}\right) & =e_{q}\left(a_{N+1} A_{-}\right)\left(\sum_{p=1}^{N} \sum_{i_{1}<\cdots<i_{p}} a_{i_{1}} \ldots a_{i_{p}} q^{A_{0}} A_{-}^{p-1}+A_{+}\right) E_{q}\left(-a_{d+1} A_{-}\right) \\
& =\left(\sum_{p=1}^{N} \sum_{i_{1}<\cdots<i_{p}} a_{i_{1}} \ldots a_{i_{p}} e_{q}\left(a_{d+1} A_{-}\right) q^{A_{0}} A_{-}^{p-1} E_{q}\left(-a_{N+1} A_{-}\right)\right. \\
& +e_{q}\left(a_{N+1} A_{-}\right) A_{+} E_{q}\left(-a_{N+1} A_{-}\right) .
\end{aligned}
$$

On the other hand

$$
e_{q}\left(a_{N+1} A_{-}\right) q^{A_{0}}=q^{A_{0}} \sum_{n=0}^{+\infty} \frac{(1-q)^{n}\left(a_{N+1} q A_{-}\right)^{n}}{(q ; q)_{n}}=q^{A_{0}} e_{q}\left(q a_{N+1} A_{-}\right) .
$$


Then we obtain

$$
e_{q}\left(a_{N+1} A_{-}\right) q^{A_{0}}=q^{A_{0}} e_{q}\left(q a_{N+1} A_{-}\right)=q^{A_{0}}\left(1-a_{N+1} A_{-}\right) e_{q}\left(a_{N+1} A_{-}\right) .
$$

Hence

$$
\prod_{i=0}^{N+1} e_{q}\left(a_{i} A_{-}\right) A_{+} \prod_{i=0}^{N+1} E_{q}\left(-a_{i} A_{-}\right)=\sigma_{1} q^{A_{0}}-\sigma_{2} q^{A_{0}} A_{-}+\cdots+(-1)^{N} \sigma_{N+1} q^{A_{0}} A_{-}^{N}+A_{+} .
$$

Then, the formula (2.18) follows from the fact that

$$
\sigma_{1} q^{A_{0}} A_{-}-\sigma_{2} q^{A_{0}} A_{-}^{2}+\cdots+(-1)^{N} \sigma_{N+1} q^{A_{0}} A_{-}^{N+1}=q^{A_{0}}\left[1-\prod_{i=0}^{N}\left(1-a_{i} A_{-}\right)\right] .
$$

The proof of (2.19) is similar to (2.18).

\section{Properties}

\subsection{Generating functions}

In this section we calculate the generating functions of the matrix coefficients $\psi_{n k}$ and $\phi_{n k}$ related to the operator $S$ given by

$$
S=E_{q}\left(\beta A_{+}\right) \prod_{i=1}^{d} e_{q}\left(a_{i} A_{-}\right) .
$$

The method is very similar to the one used in [17].

We have according to (2.9)

$$
\sum_{n=0}^{\infty} \psi_{n k} \frac{z^{n}}{\sqrt{[n]_{q} !}}=\sum_{n=0}^{\infty}<k|S| n>\frac{z^{n}}{\sqrt{[n]_{q} !}}=<k|S| \sum_{n=0}^{\infty} \frac{z^{n}}{\sqrt{[n]_{q} !}}|n>=<k| S \mid z>.
$$

Taking into account of formula (2.10), we have

$$
<k|S| z>=<k\left|E_{q}\left(\beta A_{+}\right) H_{d}\left(A_{-}\right)\right| z>=H_{d}(z)<k\left|E_{q}\left(\beta A_{+}\right) e_{q}\left(z A_{+}\right)\right| 0>,
$$

where

$$
H_{d}(z):=H_{d}\left(z, a_{1}, \ldots, a_{d}\right)=\prod_{i=1}^{d} e_{q}\left(a_{i} z\right) .
$$

On the other hand, we have successively by means of the (2.8) and $q$-binomial formula (see [5])

$$
E_{q}\left(\beta A_{+}\right) e_{q}\left(z A_{+}\right)|0\rangle=\sum_{n=0}^{\infty} \frac{\theta_{n}(z, \beta ; q)}{[n]_{q} !} A_{+}^{n}|0\rangle=\sum_{n=0}^{\infty} \frac{\theta_{n}(z, \beta ; q)}{\sqrt{[n]_{q} !}} \mid n>,
$$

and

$$
<k\left|E_{q}\left(\beta A_{+}\right) e_{q}\left(z A_{+}\right)\right| 0>=\frac{\theta_{k}(z, \beta ; q)}{\sqrt{[k]_{q} !}},
$$

where

$$
\theta_{k}(z, \beta ; q)=z^{k}(-\beta / z ; q)_{k}
$$


Hence, the matrix coefficients $\psi_{n k}$ are generated by

$$
F(z, k):=\frac{\theta_{k}(z, \beta ; q)}{\sqrt{[k]_{q} !}} H_{d}(z)=\sum_{n=0}^{\infty} \psi_{n k} \frac{z^{n}}{\sqrt{[n]_{q} !}} .
$$

\subsection{Recurrence relations}

If we apply the $q$-difference operator $D_{q}$ to each member of (3.3) and we use the following formulas

$$
\begin{gathered}
\theta_{k}(q z, \beta ; q)=q^{k}(z+\beta / q) \theta_{k-1}(z, \beta ; q), D_{q} \theta_{k}(z, \beta ; q)=[k] \theta_{k-1}(z, \beta ; q), \\
D_{q} H(z)=Q(z) H(z), Q(z)=\frac{1-\prod_{i=1}^{d}\left(1-(1-q) a_{i} z\right)}{(1-q) z} .
\end{gathered}
$$

We get

$$
\begin{aligned}
\sum_{n=1}^{\infty} \sqrt{[n]_{q}} \psi_{k n} \frac{z^{n-1}}{\sqrt{[n-1]_{q} !}} & =\frac{1}{\sqrt{(k]_{q} !}}\left([k]_{q} \theta_{k-1}(z, \beta ; q) H_{d}(q z)+\theta_{k}(z, \beta ; q) Q(z) H_{d}(z)\right) \\
& =\frac{1}{\sqrt{(k]_{q} !}}\left(\frac{[k]_{q} \theta_{k}(q z, \beta ; q)}{q^{k}(z+\beta / q)} H_{d}(q z)+\theta_{k}(z, \beta ; q) Q(z) H_{d}(z)\right), \\
& =\frac{q^{-k}[k]_{q}}{q^{k}(z+\beta / q)} F(q z, k)+\left(\sum_{i=0}^{d} \alpha_{i} z^{i}\right) F(z, k),
\end{aligned}
$$

where

$$
(z+\beta / q) Q(z)=\sum_{i=0}^{d} \alpha_{i} z^{i}
$$

Consequently

$$
\begin{aligned}
(z+\beta / q) \sum_{n=1}^{\infty} \sqrt{[n]_{q}} \psi_{n k} \frac{z^{n-1}}{\sqrt{[n-1]_{q} !}} & =q^{-k}[k]_{q} \sum_{n=0}^{\infty} q^{n} \psi_{n k} \frac{z^{n}}{\sqrt{[n]_{q} !}} \\
& +\sum_{n=0}^{\infty}\left(\sum_{i=0}^{d} \alpha_{i} \sqrt{[n]_{q} \ldots[n-i+1]_{q}} \psi_{n-i k}\right) \frac{z^{n}}{\sqrt{[n]_{q} !}} .
\end{aligned}
$$

Comparing now the coefficients of $z^{n}$, we get

Proposition 3.1. The matrix coefficients $\psi_{n k}$ satisfy the recurrence relation

$$
\frac{\beta}{q} \sqrt{[n+1]_{q}} \psi_{n+1 k}=-[n-k]_{q} \psi_{n k}+\sum_{i=0}^{d} \alpha_{i} \sqrt{[n]_{q} \ldots[n-i+1]_{q}} \psi_{n-i k} .
$$

Now, from (3.5) one can express $\psi_{n, k}$ recursively, starting from $\psi_{0 k}$. Indeed, putting $n=0$ we obtain

$$
\psi_{1 k}=\frac{q}{\beta}\left(\alpha_{0}-\frac{1-q^{-k}}{1-q}\right) \psi_{0 k}
$$

and for $n=1$, we have

$$
\psi_{2 k}=\left(\frac{q^{2}}{\beta^{2}} \frac{1-q}{1-q^{2}}\left(\alpha_{0}-\frac{1-q^{-k}}{1-q}\right)\left(\alpha_{0}-\frac{1-q^{1-k}}{1-q}\right)+\alpha_{1}\right) \psi_{0 k} .
$$


Repeating this process we arrive at the following.

Proposition 3.2. The matrix elements $\psi_{n, k}$ are expressed as

$$
\psi_{n k}=\psi_{0 k} V_{n}^{\left(a_{1}, \ldots, a_{d}\right)}\left(q^{-k}\right)
$$

where $V_{n}^{\left(a_{1}, \ldots, a_{d}\right)}\left(q^{-k}\right)$ is a polynomial of degree $n$ in $q^{-k}$ and satisfying the recurrence relation of $\operatorname{order}(d+1)$

$$
\frac{\beta}{q} \sqrt{[n+1]_{q}} V_{n+1}^{\left(a_{1}, \ldots, a_{d}\right)}\left(q^{-k}\right)=-[n-k]_{q} V_{n}^{\left(a_{1}, \ldots, a_{d}\right)}\left(q^{-k}\right)+\sum_{i=0}^{d} \alpha_{i} \sqrt{[n]_{q} \ldots[n-i+1]_{q}} V_{n-i}^{\left(a_{1}, \ldots, a_{d}\right)}\left(q^{-k}\right),
$$

with initial conditions

$$
V_{0}^{\left(a_{1}, \ldots, a_{d}\right)}\left(q^{-k}\right)=1, V_{n}^{\left(a_{1}, \ldots, a_{d}\right)}\left(q^{-k}\right)=0, n<0 .
$$

Consequently $\left\{V_{n}^{\left(a_{1}, \ldots, a_{d}\right)}\left(q^{-k}\right)\right\}_{n \geq 0}$ is $d$-orthogonal.

The associated monic polynomial $\widetilde{V}_{n}^{\left(a_{1}, \ldots, a_{d}\right)}\left(q^{-k}\right)$ is defined by

$$
\widetilde{V}_{n}^{\left(a_{1}, \ldots, a_{d}\right)}\left(q^{-k}\right)=q^{-\left(\begin{array}{l}
n \\
2
\end{array}\right)} \beta^{n}(1-q)^{n} \sqrt{[n]_{q} !} V_{n}^{\left(a_{1}, \ldots, a_{d}\right)}\left(q^{-k}\right)_{n} .
$$

The polynomial $\widetilde{V}_{n}^{\left(a_{1}, \ldots, a_{d}\right)}$ is generated by

$$
\sum_{n=0}^{\infty}(-1)^{n} \frac{\widetilde{V}_{n}^{\left(a_{1}, \ldots, a_{d}\right)}\left(q^{-k}\right)}{(q ; q)_{n}} z^{n}=\theta_{k}(z) H_{d}(z) .
$$

\subsection{Orthogonality relations}

Proposition 3.3. The matrix coefficients $\phi_{n k}$ satisfy the difference equation

$$
-[n-k]_{q} \phi_{n k}=\beta \phi_{n-1 k}-\sum_{i=0}^{d} \alpha_{i} q^{-i} \sqrt{[n+1]_{q} \ldots[n+i]_{q}} \phi_{n+i k} .
$$

Proof. From the bi-orthogonality relations (2.13) and the generating function (3.3) the matrix coefficients $\phi_{n k}$ have the following generating function

$$
G(z, k):=\frac{z^{n}}{\sqrt{[n]_{q} !} H_{d}(z)}=\sum_{k=0}^{\infty} \phi_{k n} \frac{\theta_{k}(z, \beta ; q)}{\sqrt{[k]_{q} !}} .
$$

Applying the operator $D_{q}$ to each members of (3.6) we obtain

$$
\sum_{k=1}^{\infty}[k]_{q} \phi_{n k} \frac{\theta_{k-1}(z)}{\sqrt{[k]_{q} !}}=\frac{[n]_{q} z^{n-1}}{\sqrt{[n]_{q} !} H_{d}(q z)}-\frac{z^{n}}{\sqrt{[n]_{q} ! H_{d}(z)}} Q(z) .
$$

So that

$$
\begin{aligned}
\sum_{k=1}^{\infty} q^{-k}[k]_{q} \phi_{n k} \frac{\theta_{k}(q z)}{\sqrt{[k]_{q} !}} & =\beta q^{-n} \sqrt{[n]_{q}} \frac{(q z)^{n-1}}{\sqrt{[n-1]_{q} ! H_{d}(q z)}}+\frac{[n]_{q} z^{n}}{\sqrt{[n]_{q} ! H_{d}(q z)}} \\
& -\frac{1}{\sqrt{[n]_{q} !}} \sum_{i=0}^{d} \alpha_{i} \frac{z^{n+i}}{H_{d}(q z)} .
\end{aligned}
$$

The result is finished by comparing the coefficients of $\theta_{k}(q z)$ in each members of (3.7). 
If $d>1$ it is possible to express $\phi_{n k}$ in terms of polynomials of argument $q^{-k}$. According to the above proposition, the coefficient $\phi_{n k}$ can be expressed as

$$
\phi_{n k}=\sum_{i=0}^{d-1} \phi_{i k} R_{n}^{(i)}\left(q^{-k}\right),
$$

where $R_{n}^{(i)}\left(q^{(-k)}\right.$ are polynomials of argument $q^{-k}$. The degrees of these polynomials depend on $n$ in the following manner. Assume that $n=d j+r$ where $r=0, \ldots, d-1$. Then

$$
\operatorname{deg} R_{n}^{(i)}=j \text { if } i \leq r, \operatorname{deg} R_{n}^{(i)}=j-1 \text { if } i>r .
$$

In connection with the above result we introduce the functionals vector $\left(L_{1}, L_{2}, \ldots, L_{d-1}\right)$ defined by

$$
L_{i}(f(x))=\sum_{k=0}^{\infty} f\left(q^{-k}\right) q^{\left(\begin{array}{c}
k \\
2
\end{array}\right)} \frac{\beta^{k}}{\sqrt{[k]_{q} !}} \phi_{i k}
$$

Then we have the following.

Proposition 3.4. The system of polynomials $\left\{P_{n}(x)\right\}_{n \in \mathbb{N}}$ satisfies the following vector orthogonality relation

$$
\begin{aligned}
& L_{i}\left(x^{m} \hat{P}_{n}(x)\right)=0, \quad n \geq m d+i+1, i=0, \ldots, d-1, \\
& L_{i}\left(x^{m} \hat{P}_{n}(x)\right) \neq 0, \quad n=m d+i, \quad i=0, \ldots, d-1 .
\end{aligned}
$$

Proof. Relations (3.8) and (3.9) are direct consequence of (2.13).

\section{Explicit expression of the $d$-OPS of $q$-Charlier type.}

The Al-Salam Carlitz II polynomials $V_{n}^{(a)}(x ; q)$ are defined by [12]

$$
V_{n}^{(a)}(x ; q)=(-a)^{n} q^{-\left(\begin{array}{c}
n \\
2
\end{array}\right)}{ }_{2} \phi_{0}\left(\begin{array}{c}
q^{-n}, x \\
-
\end{array} \mid q, \frac{q^{n}}{a}\right) .
$$

The polynomial $y(x)=V_{n}^{(a)}(x ; q)$ is an eigenfunction of the following second order $q$-difference operator

$$
(1-x)(a-x) y(q x)-[(1-x)(a-x)+a q] y(x)+a q y\left(q^{-1} x\right)=-\left(1-q^{n}\right) x^{2} y(x) .
$$

The Al-Salam Carlitz II polynomials are closely related to the $q$-Charlier polynomials [12]

$$
C_{n}\left(q^{-k} \mid q\right)=V_{n}\left(q^{-k} \mid q\right) .
$$

The three-term recurrence relation for the polynomials (4.1) is as follows (see [12])

$$
x V_{n}^{(a)}(x ; q)=V_{n+1}^{(a)}(x ; q)+(a+1) q^{-n} V_{n}^{(a)}(x ; q)+a q^{-2 n+1}\left(1-q^{n}\right) V_{n-1}^{(a)}(x ; q) .
$$

In this section we calculate the matrix coefficients $\psi_{n k}$ and $\phi_{n k}$ associated to the operator $S$ given by

$$
S=E_{q}\left(\beta A_{+}\right) \prod_{i=1}^{d} e_{q}\left(a_{i} A_{-}\right),
$$

in terms of the By means of technic based on the notion of a generating function, we express in this section the matrix elements $\psi_{n k}$ in terms of a $d$-OPS where $V_{n}\left(q^{-k} \mid q\right)$ are the Al-Salam Carlitz II 
polynomials.

Let $\omega=e^{2 i \pi / d}, a \in \mathbb{C}$ and we suppose for $i=0,1, \ldots, d-1, a_{i}=a \omega^{i}$. From [5, I.30], we have

$$
\left(a^{d} ; q^{d}\right)_{n}=\left(a, a \omega, \ldots, a \omega^{d-1} ; q\right)_{n} .
$$

If we let $n \rightarrow \infty$ in (4.3), we get

$$
\prod_{k=0}^{d-1} e_{q}\left(a z \omega^{k}\right)=e_{q^{d}}\left(a^{d} z^{d}\right)
$$

Hence the operator $S$ becomes

$$
S=E_{q}\left(\beta A_{+}\right) e_{q^{d}}\left(a^{d} A_{-}^{d}\right)
$$

We denote by

$$
V_{n}^{(a, d)}(x)=V_{n}^{\left(a, a \omega, \ldots, a \omega^{d-1}\right)}(x) .
$$

Now, by means of technic based on the notion of a generating function, we express the matrix elements $\psi_{n k}$ in terms of a $d$-OPS of Al-Salam Carlitz II polynomials evaluate the matrix coefficients $\phi_{n k}$ in terms of basic hypergeometric series. Expanding $\left.e_{q}\left(q^{-k} z\right)\right)^{-1}$ and $e_{q^{d}}\left(a z^{d}\right)$ in terms of $z^{n}$, we find

$$
\sum_{n=0}^{\infty}(-1)^{n} \frac{V_{n}^{(a, d)}\left(q^{-k}\right)}{(q ; q)_{n}} z^{n}=\sum_{n=0}^{\infty}\left(\sum_{i=0}^{[n / d]} \frac{a^{i}(1-q)^{n-i d}\left(1-q^{d}\right)^{i}\left(q^{-k} ; q\right)_{n-i d}}{\left(q^{d} ; q^{d}\right)_{i}(q ; q)_{n-i d}}\right) z^{n} .
$$

Consequently

$$
V_{n}^{(a, d)}\left(q^{-k}\right)=(-1)^{n}(q ; q)_{n}=\sum_{i=0}^{[n / d]} \frac{a^{i}(1-q)^{n-i d}\left(1-q^{d}\right)^{i}\left(q^{-k} ; q\right)_{n-i d}}{\left(q^{d} ; q^{d}\right)_{i}(q ; q)_{n-i d}} .
$$

According to the following identity

$$
\frac{\left(q^{-k} ; q\right)_{n-i d}}{(q ; q)_{n-i d}}=q^{-i k d} \frac{\left(q^{-k} ; q\right)_{n}}{(q ; q)_{n}} \frac{\prod_{j=1}^{d}\left(q^{-1-n+j} ; q^{d}\right)_{i}}{\prod_{j=1}^{d}\left(q^{k-n+j} ; q^{d}\right)_{i}},
$$

we obtain

$$
V_{n}^{(a, d)}\left(q^{-k}\right)=(-1)^{n}\left(q^{-k} ; q\right)_{n}(1-q)^{n} \sum_{i=0}^{\infty} \frac{\prod_{j=1}^{d}\left(q^{-1-n+j} ; q^{d}\right)_{i}}{\prod_{j=1}^{d}\left(q^{k-n+j} ; q^{d}\right)_{i}} \frac{a^{i}\left(1-q^{d}\right)^{i} q^{-i k d}}{\left(q^{d} ; q^{d}\right)_{i}(1-q)^{i d}}
$$

Finally

$$
V_{n}^{(a, d)}\left(q^{-k}\right)=(-1)^{n}\left(q^{-k} ; q\right)_{n}(1-q)^{n}{ }_{d+1} \varphi_{d}\left(\begin{array}{c}
\Delta\left(-n, d ; q^{d}\right), 0 \\
\Delta\left(-n, d ; q^{d}\right)
\end{array} \mid q^{d} ; \frac{a\left(1-q^{d}\right)^{i} q^{-k d}}{(1-q)^{d}}\right),
$$

where

$$
\Delta(\lambda, m ; q)=q^{\lambda / m}, q^{(\lambda+1) / m}, \ldots, q^{(\lambda+m-1) / m} .
$$




\subsection{Lowering and raising operators}

According to Proposition 2.1 and Proposition 2.2, we get

$$
S A_{-}=\left(A_{-}-\beta q^{A_{0}}\right) S .
$$

Then

$$
\sqrt{[n]_{q}} \psi_{n-1, k}=\left\langle k\left|S A_{-}\right| n\right\rangle=\left\langle k\left|\left(A_{-}-\beta q^{A_{0}}\right) S\right| n\right\rangle=\left\langle k\left|A_{-} S\right| n\right\rangle-\beta\left\langle k\left|q^{A_{0}} S\right| n\right\rangle
$$

and

$$
\sqrt{[n]_{q}} \psi_{n-1, k}=\sqrt{[k+1]_{q}} \psi_{n, k+1}-\beta q^{k} \psi_{n k}
$$

Recall that

$$
\psi_{0 k}=<k|S| 0>=<k\left|E_{q}\left(\beta A_{+}\right)\right| 0>=<k \mid \beta>=\frac{\beta^{k} q^{k(k-1) / 2}}{\sqrt{[k]_{q} !}} .
$$

Dividing the two members of (4.5) by $\psi_{0 k}$ we get

$$
\beta^{k} q^{k}\left(V_{n}^{\left(a_{1}, \ldots, a_{d}\right)}\left(q^{-k-1}\right)\left(q^{-(k+1)}\right)-V_{n}^{\left(a_{1}, \ldots, a_{d}\right)}\left(q^{-k}\right)\right)=\sqrt{[n]_{q}} V_{n-1}^{\left(a_{1}, \ldots, a_{d}\right)}\left(q^{-k}\right) .
$$

Since

$$
\widetilde{V}_{n}^{\left(a_{1}, \ldots, a_{d}\right)}\left(q^{-k}\right)=q^{-\left(\begin{array}{c}
n \\
2
\end{array}\right)} \beta^{n}(1-q)^{n} \sqrt{[n]_{q} !} V_{n}^{\left(a_{1}, \ldots, a_{d}\right)}\left(q^{-k}\right)_{n} .
$$

So that

$$
q^{k}\left(\widetilde{V}_{n}^{\left(a_{1}, \ldots, a_{d}\right)}\left(q^{-k-1}\right)-\widetilde{V}_{n}^{\left(a_{1}, \ldots, a_{d}\right)}\left(q^{-k}\right)\right)=\left(q^{n}-1\right) \widetilde{V}_{n-1}^{\left(a_{1}, \ldots, a_{d}\right)}\left(q^{-k}\right) .
$$

On other words

$$
\left(D_{q^{-1}} \widetilde{V}_{n}^{\left(a_{1}, \ldots, a_{d}\right)}\right)\left(q^{-k}\right)=[n]_{q} \widetilde{V}_{n}^{\left(a_{1}, \ldots, a_{d}\right)}\left(q^{-k}\right) .
$$

From Proposition 2.2 and Proposition 2.3, we can write

$$
\begin{aligned}
S A_{+} S^{-1} & =E_{q}\left(\beta A_{+}\right)\left(A_{+} q^{A_{0}} Q\left(A_{-}\right)\right) e_{q}\left(-\beta A_{+}\right) \\
& =A_{+}+\left(1+(1-q) \beta A_{+}\right) q^{A_{0}} Q\left(A_{-}-(1-q) \beta q^{A_{0}}\right) .
\end{aligned}
$$

Hence

$$
S A_{+}=\left(A_{+}+\left(1+(1-q) \beta A_{+}\right) q^{A_{0}}\right)\left(A_{-}-(1-q) \beta q^{A_{0}}\right)^{d-1} S .
$$

The operator $A_{-}$and $q^{A_{0}}$ satisfy the $q$-commutation relation

$$
A_{-} q^{A_{0}}=q q^{A_{0}} A_{-} .
$$

Then from the well know $q$-binomial Newton formula for $q$-commuting variables (see [14]) we get

$$
\left(A_{-}-(1-q) \beta q^{A_{0}}\right)^{d-1}=\sum_{s=0}^{d-1}\left[\begin{array}{c}
d-1 \\
s
\end{array}\right]_{q}(-(1-q) \beta)^{d-s-1} q^{(d-s-1) A_{0}} A_{-}^{s} .
$$


From (4.6), we have

$$
\begin{aligned}
\sqrt{[n+1]_{q}} \psi_{n+1 k} & =<k\left|S A_{+}\right| n> \\
& =<k\left|A_{+}+\left(1+(1-q) \beta A_{+}\right) q^{A_{0}} Q\left(A_{-}-(1-q) \beta q^{A_{0}}\right)\right| n> \\
& =\sqrt{[n+1]_{q}}\left(1-(-(1-q) \beta)^{d-1} q^{k d}\right) \psi_{n k+1}+\sum_{s=0}^{d-1}\left[\begin{array}{c}
d-1 \\
s
\end{array}\right]_{q}(-(1-q) \beta)^{d-s-1} \\
& \times q^{(d-s-1) k} \sqrt{\frac{[k]_{q} !}{[k-s]_{q} !}} \psi_{n k-s}+\sum_{s=0}^{d-2}\left[\begin{array}{c}
d-1 \\
s+1
\end{array}\right]_{q}(-(1-q) \beta)^{d-s-1} q^{(d-s-1) k} \\
& \times[k+1]_{q} \sqrt{\frac{[k]_{q} !}{[k-s]_{q} !}} \psi_{n k-s} .
\end{aligned}
$$

Hence

$$
\begin{aligned}
\sqrt{[n+1]_{q}} \psi_{n+1 k} & =\sqrt{[n+1]_{q}}\left(1-(-(1-q) \beta)^{d-1}\right) q^{k d} \psi_{n k+1}+\sqrt{\frac{[k]_{q} !}{[k-d-1]_{q} !}} \\
& \times \psi_{n k-d+1}+\sum_{s=0}^{d-1}\left[\begin{array}{c}
d-1 \\
s
\end{array}\right]_{q}(-(1-q) \beta)^{d-s-1} q^{(d-s-1) k} \sqrt{\frac{[k]_{q} !}{[k-s]_{q} !}} \\
& \times\left(1+\frac{[d-s-2]_{q}[k+1]_{q}}{[s+1]_{q}}\right) \psi_{n k-s} .
\end{aligned}
$$

Henceforth

$$
\begin{aligned}
\sqrt{[n+1]_{q}} V_{n+1}^{(a d)}\left(q^{-k}\right) & =\sqrt{[n+1]_{q}}\left(1-(-(1-q) \beta)^{d-1}\right) q^{k d} \\
& \times V_{n}^{(a d)}\left(q^{-k-1}\right)+\sqrt{\frac{[k]_{q} !}{[k-d-1]_{q} !}} V_{n}^{(a d)}\left(q^{-k+d-1}\right) \\
& +\sum_{s=0}^{d-1}\left[\begin{array}{c}
d-1 \\
s
\end{array}\right]_{q}(-(1-q) \beta)^{d-s-1} q^{(d-s-1) k} \sqrt{\frac{[k]_{q} !}{[k-s]_{q} !}} \\
& \times\left(1+\frac{[d-s-2]_{q}[k+1]_{q}}{[s+1]_{q}}\right) V_{n}^{(a d)}\left(q^{-k+s}\right) .
\end{aligned}
$$

Let introduce the operator $R_{q}$

$$
\begin{aligned}
R_{q} & =\sqrt{[n+1]_{q}}\left(1-(-(1-q) \beta)^{d-1}\right) q^{k d} \\
& \times T_{q^{-1}}^{k+1}+\sqrt{\frac{[k]_{q} !}{[k-d-1]_{q} !}} T_{q^{-1}}^{k-d+1} \\
& +\sum_{s=0}^{d-1}\left[\begin{array}{c}
d-1 \\
s
\end{array}\right]_{q}(-(1-q) \beta)^{d-s-1} q^{(d-s-1) k} \sqrt{\frac{[k]_{q} !}{[k-s]_{q} !}} \\
& \times\left(1+\frac{[d-s-2]_{q}[k+1]_{q}}{[s+1]_{q}}\right) T_{q^{-1}}^{k-s} .
\end{aligned}
$$


Here $T_{q^{-1}}$ is the $q$-shift operator defined by $\left(T_{q^{-1}} P\right)(x)=P\left(q^{-1} x\right)$. Then

$$
\left(R_{q} V_{n}^{(a d)}\right)\left(q^{-k}\right)=\sqrt{[n+1]_{q}} V_{n+1}^{(a d)}\left(q^{-k}\right) .
$$

Note that the operators $D_{q^{-1}}$ and $R_{q}$ satisfy the relation

$$
R_{q} D_{q^{-1}}-q D_{q^{-1}} R_{q}=1 .
$$

In order to find the dual function $\phi_{n k}$, we need the following Lemma.

Lemma 4.1. If $f(z)=\sum_{n=0}^{\infty} a_{k} \theta_{k}(z)$, then

$$
a_{k}=\frac{1}{[k]_{q} !}\left[D_{q}^{k} f(z)\right]_{z=-\beta} .
$$

If $d=1$, by Lemma 4.1 , we can write

$$
\begin{aligned}
\phi_{0 k}= & \frac{1}{\sqrt{[k]_{q} !}}\left[D_{q}^{k}\left(E_{q}(-a z)\right)\right]_{z=-\beta}=\frac{1}{\sqrt{[k]_{q} !}}(-a)^{k} q^{\left(\begin{array}{c}
k \\
2
\end{array}\right)}\left[E_{q}\left(-a z q^{k}\right)\right]_{z=-\beta} \\
= & \frac{1}{\sqrt{[k]_{q} !}}(-a)^{k} q^{\left(\begin{array}{l}
k \\
2
\end{array}\right)} E_{q}\left(\frac{-a q^{k+1}}{1-q}\right)=\frac{1}{\sqrt{[k]_{q} !}}(-a)^{k} q^{\left(\begin{array}{c}
k \\
2
\end{array}\right)} \frac{(a, q)_{\infty}}{(a q ; q)_{k}} .
\end{aligned}
$$

On the other hand,

$$
\phi_{0 k} \psi_{0 k}=\frac{a^{k} q^{k^{2}}}{(a q ; q)_{k}(q ; q)_{k}} .
$$

Consequently

$$
L_{0}\left(V_{n}^{(a)}(x) V_{m}^{(a)}(x)\right)=\sum_{k=0}^{\infty} \frac{a^{k} q^{k^{2}}}{(a q ; q)_{k}(q ; q)_{k}} V_{n}^{(a)}\left(q^{-k}\right) V_{m}^{(a)}\left(q^{-k}\right)=0, n \neq m .
$$

If $d \geq 2$, then according to Lemma 4.1, we get

$$
\begin{aligned}
& \phi_{n k}=\frac{1}{\sqrt{[k]_{q} !}}\left[D_{q}^{k}\left(\frac{z^{n}}{\sqrt{[n]_{q} !}} E_{q^{d}}\left(-a^{d} z^{d}\right)\right)\right]_{z=-\beta} \\
& =\frac{q^{n-k}\left(a^{d} ; q^{d}\right)_{\infty}}{(1-q)^{n} \sqrt{[k]_{q} ![n]_{q} !}} \sum_{i=0}^{\infty} \frac{q^{i(n+1)}\left(q^{-k} ; q\right)_{i}}{(q ; q)_{i}\left(a^{d} ; q^{d}\right)_{i}} \\
& =\frac{q^{n-k}\left(a^{d} ; q^{d}\right)_{\infty}}{(1-q)^{n} \sqrt{[k]_{q} ![n]_{q} !}} d+1 \varphi_{d}\left(\begin{array}{c}
q^{-k}, 0,0, \ldots, 0 \\
a, a \omega, \ldots, a \omega^{d-1}
\end{array} \mid q ; q^{n+1}\right) \text {. }
\end{aligned}
$$

Acknowledgments. This research project is supported by King Saud University, Riyadh, DSFP, through the grant MATH 01. 


\section{References}

[1] Y. Ben Cheikh and A. Ouni, Some generalized hypergeometric $d$-orthogonal polynomial sets, Journal of Mathematical Analysis and Applications, 343(1):464-478, 2008.

[2] Y. Ben Cheikh and A. Zaghouani, Some discrete $d$-orthogonal polynomial sets, Journal of Computational and Applied Mathematics, 156(2):253-263, 2003.

[3] J. Dixmier, Sur les algèbres de Weyl, Bull. Soc. Math. France 96, 209 (1968). 10.

[4] K. Douak, The relation of the $d$-orthogonals to the Appell polynomials, J. Comput. Appl. Math. 70, 279 (1996).

[5] G. Gasper and M. Rahman, Basic Hypergeometric Series, 2nd Edition, (2004), Encyclopedia of Mathematics and Its Applications, 96, Cambridge University Press, Cambridge.

[6] Ya. I. Granovskii, and A. S. Zhedanov, Orthogonal polynomials on Lie algebras, Izv. Vyssh. Uchebn. Zaved. Fiz. 29, 60 (1986) (in Russian); Sov. Phys. J. 29, 387 (1986).

[7] J. van Iseghem, Laplace transform inversion and padé-type approximants, Applied Numerical Mathematics, 3(6):529-538, November 1987.

[8] M. E. H. Ismail, Classical and Quantum Orthogonal Polynomials in one Variable, Cambridge University Press, paperback edition, Cambridge, 2009.

[9] C. Kassel, Quantum Groups, Springer-Verlag, Berlin, 1995.

[10] A. Kempf and S. Majid, Algebraic $q$-Integration and Fourier Theory on Quantum and Braided Spaces, J.Math.Phys. 35 (1994) 6802-6837.

[11] A. U. Klimyk, and W. Schempp, Classical and quantum Heisenberg groups, their representations and applications, Acta Appl. Math. 45, 143 (1996).

[12] R. Koekoek, and R. F. Swarttouw, The Askey scheme of hypergeometric orthogonal polynomials and its $q$-analogue, Faculty of Technical Mathematics and Informatics, Delft University of Technology Report No. 94-05, 1994.

[13] T. H. Koornwinder, Basic Hypergeometric Functions, Compact Quantum Groups and $q$-Special Functions, Representations of Lie Groups and Quantum Groups (Pitman Research Notes 311), V. Baldoni and M. A. Picardello, eds.), Longman Scientific \& Technical, Essex, UK, 1994, pp. 46-128.

[14] T. H. Koornwinder, Special functions and $q$-commuting variables, Fields Institute Communications 14 (1997) 131-166.

[15] P. Maroni, L'orthogonalité et les récurrences de polynômes d'ordre supérieur à deux, Annales de la faculté des sciences de Toulouse, 10(1):105-139, January 1989.

[16] A. Perelomov, Generalized Coherent States and Their Applications, Springer-Verlag Berlin 1986.

[17] L. Vinet and A. Zhedanov, Automorphisms of the Heisenberg-Weyl algebra and $d$-orthogonal polynomials. Journal of Mathematical Physics, 50(3) 1-19, 2009. 